\title{
The Non Linear Controller for Terminal Voltage and Power Angle of Power Systems
}

\author{
Moulay Fatima ${ }^{1}$, Ramdani Youcef ${ }^{2}$, Habbati Assia $^{2}$, Hamdaoui \\ Habib $^{2}$, Bendaoud Abdelber ${ }^{3}$
}

${ }^{1}$ LMSR Laboratory; Djilali Liabes University of Sidi Bel-Abbes; Tlemcen street, Sidi Bel-Abbes (22000), Algeria. E-mail: babdelber@univ-sba.dz

${ }^{2}$ IRECOM Laboratory; Djilali Liabes University of Sidi Bel-Abbes; Algeria Tlemcen street, Sidi Bel-Abbes (22000), Algeria. E-mail: ramdani@univ-sba.dz

${ }^{3}$ APELEC Laboratory; Djilali Liabes University of Sidi Bel-Abbes; Algeria Tlemcen street, Sidi Bel-Abbes (22000), Algeria. E-mail: babdelber@gmail.com

\begin{abstract}
The purposed work is the design of a nonlinear voltage and rotor angle controller to improve the stability properties of a system which comprises a synchronous generator connected to an infinite bus and hydraulic turbine. The controller is based on the feedback linearization scheme. The advantage of this systematic nonlinear controller design is that the control system is liberalized in a wider domain. A linear control can then be used to stabilize the linear system. The performances of the linear voltage and rotor angle regulators are compared with those of the standard linear voltage and speed regulators in the presence of short circuits at the generators. The infinite bus and the nonlinear regulator improved considerably the stability and the transient dynamic performance of the generator.
\end{abstract}

Keywords: Nonlinear control; hydraulic turbine; synchronous generator; voltage regulation; rotor angle stability; feedback linearization

\section{Introduction}

The control equipment of synchronous generators, such as an automatic voltage regulator, a speed governor and a power system stabilizer, are normally designed for a liberalized model of a power system around a given operating conditions. Therefore, when the operating condition or the network configuration changes widely, it may become less effective because of the non linearity of the power system. This became a very important problem in power systems. To solve this problem and to realize efficient control performance, many advanced studies have been done on generator control systems, such as adaptive generator controllers based on linear control theory[1] and self-turning power system stabilizers based 
on adaptive control theory. The technique of input-output feedback linearization is now well known because it gives a good solution for tracking control problems [2]. This later one has been successfully applied to the control of AC motors and synchronous generators. The main goal of this paper is to investigate the application of a nonlinear control technic to a detailed multi-input, multi-output nonlinear model of a power system in order to improve both, its stability and damping properties even under large and sudden disturbances and to insure good post-fault voltage regulation [3].

\section{Model of the System}

The generator is connected to an infinite bus through a transmission line having resistance $\mathrm{R}_{\mathrm{e}}$ and inductance $\mathrm{L}_{\mathrm{e}}$. We applied classical Park's transformation, and the model takes into account both field effects and damper-winding effects introduced by the different rotors circuits as follows:

In the axe $d$ :

$\left[\begin{array}{c}i_{d} \\ i_{f d} \\ i_{k d}\end{array}\right]=\frac{\left[\begin{array}{ccc}-\left(L_{f d} \cdot L_{k d}-L_{m d}^{2}\right) & \left(L_{m d} \cdot L_{k d}-L_{m d}^{2}\right) & \left(L_{f d} \cdot L_{m d}-L_{m d}^{2}\right) \\ -\left(L_{m d} \cdot L_{k d}-L_{m d}^{2}\right) & \left(L_{d} \cdot L_{k d}-L_{m d}^{2}\right) & -\left(L_{d} \cdot L_{m d}-L_{m d}^{2}\right) \\ -\left(L_{f d} \cdot L_{m d}-L_{m d}^{2}\right) & \left(L_{d} \cdot L_{m d}-L_{m d}^{2}\right) & \left(L_{f d} \cdot L_{d}-L_{m d}^{2}\right)\end{array}\right]}{L_{d} \cdot L_{f d} \cdot L_{k d}-L_{m d}^{2} \cdot\left(L_{d}+L_{f d}+L_{k d}\right)+2 \cdot L_{m d}^{3}}\left[\begin{array}{c}\Phi_{d} \\ \Phi_{f d} \\ \Phi_{k d}\end{array}\right]$

In the axe q:

$$
\begin{aligned}
& {\left[\begin{array}{c}
i_{q} \\
i_{k q}
\end{array}\right]=\frac{\left[\begin{array}{cc}
-L_{k q} & L_{m q} \\
-L_{m q} & L_{q}
\end{array}\right]}{L_{q} \cdot L_{k q}-L_{m q}^{2}} \cdot\left[\begin{array}{c}
\Phi_{q} \\
\Phi_{k q}
\end{array}\right]} \\
& \frac{d\left(\Phi_{q}\right)}{d t}=v_{q}+R_{s} \cdot i_{q}-\omega \cdot \Phi_{d} \\
& \frac{d\left(\Phi_{d}\right)}{d t}=v_{d}+R_{s} \cdot i_{d}+\omega \cdot \Phi_{q} \\
& \frac{d\left(\Phi_{k d}\right)}{d t}=-R_{k d} \cdot i_{k d} \\
& \frac{d\left(\Phi_{k q}\right)}{d t}=-R_{k q} \cdot i_{k q} \\
& \frac{d\left(\Phi_{f d}\right)}{d t}=v_{f d}-R_{f d} \cdot i_{f d}
\end{aligned}
$$

Where: $v_{d}(t), v_{q}(t)$ and $v_{f d}(t)$ are respectively the direct-axis, quadrature-axis terminal voltages, and excitation control input. 
And :

$\mathrm{i}_{\mathrm{d}}(\mathrm{t}), \mathrm{i}_{\mathrm{q}}(\mathrm{t})$ : direct-axis and quadrature-axis currents

$\mathrm{i}_{\mathrm{kd}}(\mathrm{t}), \mathrm{i}_{\mathrm{kq}}(\mathrm{t})$ : direct-axis and quadrature-axis damper windings currents

$\mathrm{i}_{\mathrm{fd}}(\mathrm{t})$ : field winding current

$\mathrm{R}_{\mathrm{s}}, \mathrm{R}_{\mathrm{kd}}, \mathrm{R}_{\mathrm{kq}}$ and $\mathrm{R}_{\mathrm{fd}}$ : stator resistance, damper windings resistances, and field resistance

$\mathrm{L}_{\mathrm{d}}, \mathrm{L}_{\mathrm{q}}, \mathrm{L}_{\mathrm{fd}}$ : direct and quadrature self-inductances, rotor self-inductance

$\mathrm{L}_{\mathrm{kd}}, \mathrm{L}_{\mathrm{kq}}$ : direct and quadrature damper windings self-inductances

$\mathrm{L}_{\mathrm{md}}$ inductance, $\mathrm{L}_{\mathrm{mq}}$ : direct and quadrature magnetizing.

The mechanical dynamics of the machine rotor are given in these equations:

$2 H \omega_{R} \frac{d \omega}{d t}=T_{m}-T_{e}-T_{f}$

$T_{m}=\frac{P_{m}}{\omega}$

$T_{e}=\left(L_{q}-L_{d}\right) i_{d} i_{q}+L_{m d} i_{f d} i_{q}+L_{m d} i_{k d} i_{q}-L_{m q} i_{d} i_{k q}$

$T_{f}=F . \omega$

$H=\frac{\omega_{R}}{S_{R}}$

$2 H . \omega_{R} \frac{d \omega}{d t}=\frac{P_{m}}{\omega}-\left(L_{q}-L_{d}\right) i_{d} i_{q}-L_{m d} i_{f d} i_{q}-L_{m d} i_{k d} i_{q}+L_{m q} i_{d} i_{k q}-F . \omega$

$\frac{d \delta}{d t}=(\omega-1)$

Where: $T_{e}$ is the electromagnetic torque, and $T_{f}$ is the resistant torque

$\mathrm{T}_{\mathrm{m}}$ : mechanical torque, $\mathrm{H}:$ inertia constant, $\omega:$ speed of the generator, $\mathrm{F}$ : the coefficient of friction, $\delta$ : power angle of the generator $\mathrm{P}_{\mathrm{m}}, \mathrm{S}_{\mathrm{R}}$ mechanic power and nominal power.

The model of the synchronous machine connected to an infinite bus, the d-q terminal voltages $\mathrm{v}_{\mathrm{d}}$ and $\mathrm{v}_{\mathrm{q}}$ are given by the load equations. After using the Park's transformation, we can write:

$\left[\begin{array}{l}v_{q} \\ v_{d} \\ v_{o}\end{array}\right]=R_{e}\left[\begin{array}{l}i_{q} \\ i_{d} \\ i_{o}\end{array}\right]+L_{e} \frac{d}{d t}\left[\begin{array}{c}i_{q} \\ i_{d} \\ i_{o}\end{array}\right]-\omega \cdot L_{e}\left[\begin{array}{c}-i_{d} \\ i_{q} \\ 0\end{array}\right]+v\left[\begin{array}{c}\sin (-\delta+a) \\ \cos (-\delta+a) \\ 0\end{array}\right]$

Where $v$ is the rms value of the bus voltage and a is its phase angle. 


\subsection{Model of the Hydraulic Turbine}

The hydraulic turbine can be modeled by:

$$
\begin{aligned}
& \frac{d q}{d t}=\frac{1}{T_{w}}\left(1-\frac{q^{2}}{A_{t}^{2} g^{2}}\right) \\
& P_{m}=\frac{q^{3}}{A_{t}^{2} \cdot g^{2}}
\end{aligned}
$$

Where: $q, g, T_{w}$ and $A_{t}$ : flow in the conduit, gate opening, water time constant, constant proportionality factor

$$
\begin{aligned}
& \frac{d q}{d t}=\frac{-2}{T_{w} G_{0}} q+\frac{2 A_{t}}{T_{w} G_{0}} g \\
& P=3 q-2 A_{t} g
\end{aligned}
$$

\subsection{Model of the Servomotor}

The servomotor is modelized by the block diagram of Fig. 1:

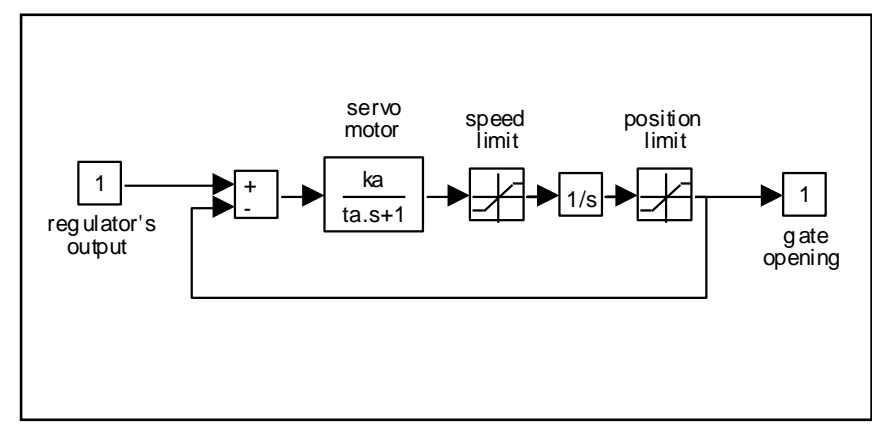

Figure 1

Model of the servomotor

Where $k_{a}$ and $t_{a}$ successively the gate servomotor gain and time constant the regulator of voltage has as a role to maintain the final voltage of the generator in its face value of 1 per unit (p.u). It uses the seven state variables of the controlled system, which is the generator on the infinite bar, the measure of mechanical potency and reference of voltage. They assume of course that they can have state variables of the system. Otherwise, the non measurable variables can be estimated by observation.

The generator linked to an infinite network is a system of order 7 state variables of which are currents $i_{d}, i_{q}, i_{\text {fd }}, i_{k d}$ and $i_{k q}$, speed and internal angle. The input of the control is the voltage. 
State equations of our system in p.u. in plan d-q are:

$$
\begin{aligned}
& \frac{d x}{d t}=F(x)+G(x) * u_{1}
\end{aligned}
$$

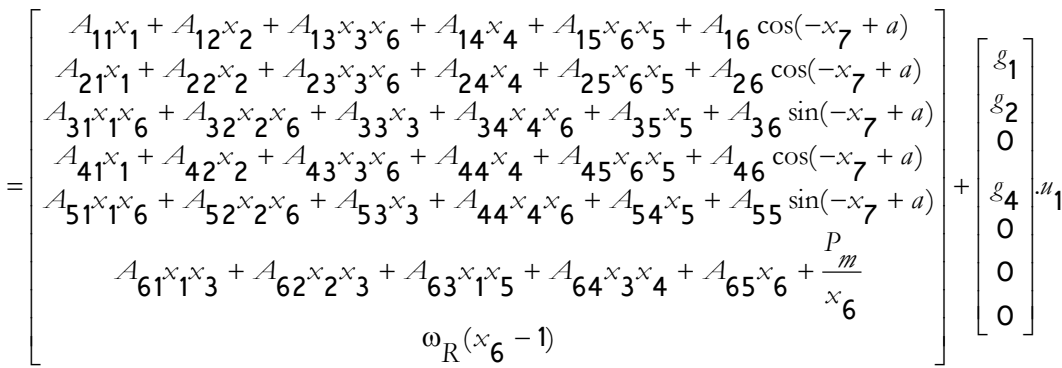

$$
\begin{aligned}
& y=v_{t}(x)=\sqrt{v_{d}^{2}(x)+v_{q}^{2}(x)} \\
& v_{d}(x)=c_{11} x_{1}+c_{12} x_{2}+c_{13} x_{3} x_{6}+ \\
& c_{14} x_{4}+c_{15^{x} 5^{x}}+c_{16} \cos \left(-x_{7}+a\right) \\
& v_{q}(x)=c_{21} x_{1} x_{6}+c_{22} x_{2} x_{6}+c_{23} x_{3}+ \\
& c_{24} x_{4} x_{6}+c_{25} x_{5}+c_{26} \sin \left(-x_{7}+a\right) \\
& x=\left[\begin{array}{lllllll}
x_{1} & x_{2} & x_{3} & x_{4} & x_{5} & x_{6} & x_{7}
\end{array}\right]^{T}= \\
& {\left[\begin{array}{lllllll}
i_{d} & i_{f d} & i_{q} & i_{k d} & i_{k q} & \omega & \delta
\end{array}\right]^{T}}
\end{aligned}
$$

$\mathrm{P}_{\mathrm{m}}$, and $\mathrm{y}$ are the vector of state variables, $\mathrm{u}_{1}$ is the vector of control inputs. $\mathrm{A}_{\mathrm{ij}}$ and $\mathrm{C}_{\mathrm{kl}}$ are constants which depend on the generator's parameters [3].

\section{Feedback Linearization of the System}

The first output to be chosen is the terminal voltage $\mathrm{v}_{\mathrm{t}}$

$$
y=v_{t}(x)=\sqrt{v_{d}^{2}(x)+v_{q}^{2}(x)}
$$

The expressions of $\mathrm{v}_{\mathrm{d}}$ and $\mathrm{v}_{\mathrm{q}}$ as a function of the state variables can be obtained by combining equations (1) and (5) which gives equation (9)

In order to obtain the nonlinear controller $\mathrm{u}_{1}$, we compute the time derivative of the output $y$.

$$
\dot{y}=\dot{v}_{t}(x)=\frac{\partial v_{t}}{\partial x} \dot{x}=\frac{1}{v_{t}}\left(v_{d} \frac{\partial v_{d}}{\partial x}+v_{q} \frac{\partial v_{q}}{\partial x}\right)\left(F(x)+G(x) u_{1}\right)
$$




$$
\begin{aligned}
& \frac{\partial v_{d}}{\partial x}=\left[\begin{array}{llllllll}
c_{11} & c_{12} & c_{13} x_{6} & c_{14} & c_{15^{x}} x_{6} & c_{13} x_{3}+c_{15} x_{5} & c_{16} \sin \left(-x_{7}+a\right)
\end{array}\right] \\
& \frac{\partial v_{q}}{\partial x}=\left[\begin{array}{lllllll}
c_{21} x_{6} & c_{22} x_{6} & c_{23} & c_{24} x_{6} & c_{25} & c_{21} x_{1}+c_{22} x_{2}+c_{24} x_{4} & -c_{26} \cos \left(-x_{7}+a\right)
\end{array}\right] \\
& \dot{y}=a(x)+b(x) * u_{1}
\end{aligned}
$$

Where:

$$
\begin{aligned}
& a(x)=\frac{1}{v_{t}}\left(v_{d} \frac{\partial v_{d}}{\partial x}+v_{q} \frac{\partial v_{q}}{\partial x}\right) F(x) \\
& b(x)=\frac{1}{v_{t}}\left(v_{d} \frac{\partial v_{d}}{\partial x}+v_{q} \frac{\partial v_{q}}{\partial x}\right) G(x)
\end{aligned}
$$

We choose $u_{1}$ that:

$$
u_{1}=\frac{(-a(x)+v)}{b(x)}, b(x) \neq 0
$$

We obtain the linear differential equation in first order between the output and the new input.

$\dot{y}=v$

The relative degree corresponding to the output is:

$y=v_{t}$ is $r_{l}=1$

$v=-k_{t}\left(y-y_{r e f}\right)=-k_{t}\left(v_{t}-v_{r e f}\right) k_{t}>0$

$\mathrm{V}_{\text {ref }}$ is the reference to terminal voltage. So that the linear control is low, the dynamic system feedback is:

$$
\begin{aligned}
& \dot{y}+k_{t} y=k_{t} y_{r f f} \\
& \frac{y}{y_{r e f}}=\frac{v_{t}}{v_{r e f}}=\frac{1}{s^{*} \tau+1} \\
& \tau=\frac{1}{k_{t}} \quad \text { is the time constant } \\
& v=-k_{t}\left(y-y_{r f}\right)+\dot{y}_{r f}=-k_{t}\left(v_{t}-v_{r f f}\right)+\dot{v}_{r f} \quad, k_{t}>0
\end{aligned}
$$

In case the dynamic tracking error " $\mathrm{e}$ " is:

$$
\dot{e}+k_{t} e=0
$$

A judicious choice of $\mathrm{k}_{\mathrm{t}}$ is necessary to assure a good tracking. 


$$
\begin{aligned}
& \dot{x}=\left[\begin{array}{c}
\dot{x}_{1} \\
\dot{x}_{2} \\
\dot{x}_{3} \\
\dot{x}_{4}
\end{array}\right]=\left(\begin{array}{c}
\omega_{R}\left(x_{2}-1\right) \\
P_{e}+\frac{x_{3}^{3}}{\left(A_{t} x_{4}\right)^{2}} \\
\frac{1}{T_{w}}\left(1-\frac{x_{3}^{2}}{A_{t}^{2} x_{4}^{2}}\right) \\
-\frac{1}{T_{g}} x_{4}
\end{array}\right)+\left(\begin{array}{c}
0 \\
0 \\
0 \\
\frac{1}{T_{g}}
\end{array}\right) u \\
& y=\delta \\
& x=\left[\begin{array}{llll}
\delta & \omega & q & g
\end{array}\right]^{T}
\end{aligned}
$$

Successive derivations of the output give:

$$
\begin{aligned}
& \dot{y}=\dot{\delta}=\omega_{R}\left(x_{2}-1\right) \\
& \ddot{y}=\omega_{R} \dot{\omega}=\left(P_{e}+\frac{q^{3}}{A_{t}^{2} g^{2}}\right) \cdot \omega_{R} \\
& y^{(3)}=\frac{\omega_{R} q^{2}}{A_{t}^{2} g^{2}}\left(3 \dot{q}-\frac{2 q \cdot \dot{g}}{g}\right)=\frac{\omega_{R} q^{2}}{A_{t}^{2} g^{2}}\left\{\frac{3}{T_{w}}\left(1-\frac{q^{2}}{A_{t}^{2} g^{2}}\right)+\frac{2 q}{T_{g} g} g\right\}-\frac{2 \omega_{R} q^{2}}{A_{t}^{2} g^{3} T_{g}} u \\
& y^{(3)}=a(x)+b(x) u \\
& a(x)=\frac{\omega_{R} q^{2}}{A_{t}^{2} g^{2}}\left\{\frac{3}{T_{w}}\left(1-\frac{q^{2}}{A_{t}^{2} g^{2}}\right)+\frac{2 q}{T_{g}}\right\} \\
& b(x)=\frac{-2 \omega_{R} q^{2}}{A_{t}^{2} g^{3} T_{g}} \\
& \begin{array}{l}
u-a(x) \\
b(x)
\end{array} \text { if } b(x) \neq 0 \\
& y^{(3)}=v
\end{aligned}
$$

The relative degree corresponding to the output $\mathrm{y}_{2}=\delta$ is $\mathrm{r}_{2}=3$.

$v=-k_{1}\left(y-y_{\text {ref }}\right)-k_{2}\left(\dot{y}-\dot{y}_{\text {ref }}\right)-k_{3}\left(\ddot{y}-\ddot{y}_{\text {ref }}\right)=$

$-k_{1}\left(\delta-\delta_{r e f}\right)-k_{2} \omega_{R}(\omega-1)-k_{3} \omega_{R} \dot{\omega}$

$\mathrm{k}_{1}, \mathrm{k}_{2}$ and $\mathrm{k}_{3}$ are real positive numbers, $\boldsymbol{\delta}_{\mathrm{ref}}$ the reference of the rotor angle.

The implementation of control $v$ requires that stemming of the speed (acceleration) be available. It can seem problematic. Indeed, it is not always easy to have an accelerometer. However, it is necessary to point out that control $v$ is no more and no less than P.I.D., the implementation of which is very well-known and well-controlled. 
The dynamic equation of the system in a continuous closed loop is:

$$
y^{(3)}+k_{3} \ddot{y}+k_{2} \dot{y}+k_{1} y=k_{1} y_{r e f}
$$

The equation which characterises it given by:

$$
\begin{aligned}
& s^{3}+k_{3} s^{2}+k_{2} s+k_{1}=0 \\
& s^{3}+\left(p_{3}+p_{1}+p_{2}\right) s^{2}+\left(p_{2} p_{3}+p_{1} p_{2}+p_{1} p_{3}\right) s+p_{1} p_{2} p_{3}
\end{aligned}
$$

By identification, constants $\mathrm{k}_{\mathrm{i}}$ are easily determined as follows:

$$
\begin{aligned}
& k_{3}=p_{3}+p_{1}+p_{2} \\
& k_{2}=p_{2} p_{3}+p_{1} p_{2}+p_{1} p_{3} \\
& k_{1}=p_{1} p_{2} p_{3}
\end{aligned}
$$

It is necessary to note that $\mathrm{p}_{3}$ must be a negative real pole and $\mathrm{p}_{1}$ and $\mathrm{p}_{2}$ two negative or complex real poles conjugated in negative real part. A way to choose poles $\mathrm{p}_{1}, \mathrm{p}_{2}$ and $\mathrm{p}_{3}$ is to make sure that the system in a continuous closed loop acts as a system of second control, having a time of stabilization $t_{r}$ and an overtaking $d$. The third pole is then chosen 10 times as big as the real part of poles.

So:

$$
\begin{aligned}
& \xi=\sqrt{\frac{1}{1+\left(\frac{\pi}{\log (1 / d)}\right)^{2}}} \\
& \omega_{n}=\frac{5}{\xi . t_{r}} \\
& p_{1}=-\xi \omega_{n}-j \omega_{n} \sqrt{1-\xi^{2}} \\
& p_{2}=-\xi \omega_{n}+j \omega_{n} \sqrt{1-\xi^{2}} \\
& p_{3}=-10 \xi \omega_{n}
\end{aligned}
$$

\subsection{Study of the Stability of the System}

The stability of the system in a closed loop depends on the internal dynamic which is determined from the dynamic of zero. In fact, the dynamic of zero is the internal dynamic when the system's output is equal to zero. In our case, the output will be considered equal to the reference value.

The proposed method consists in proving the stability of the dynamic of zero. If it is unstable, it should be offered a variant of the linear control which stabilizes the system in closed loop. 


\subsection{Study of Internal Dynamics}

Since the relating degree of the system is 3 , so, internal dynamic is first rate. The variable of this dynamic must verify the next equation:

$L_{G(x)} \cdot \eta(x)=0 \Rightarrow \frac{1}{T_{g}} \frac{\partial \eta}{\partial g}=0$

And it must also be linearly independent of external variables $z_{i}(i=1, . ., 0)$.

A resolution is to choose:

$\eta=q$

Therefore expressions of new state variables according to previous assumptions are:

$$
\begin{aligned}
& z_{1}=\delta \\
& z_{2}=\omega_{R}(\omega-1) \\
& z_{3}=\omega_{R}\left(P_{e}+\frac{q^{3}}{A_{t}^{2} g^{2}}\right)
\end{aligned}
$$

$\eta=q$

Then, they lead to deduce easily initial variables with respect to the new state variables.

$$
\begin{aligned}
& \delta=z_{1} \\
& \omega=1+\frac{z_{2}}{\omega_{R}} \\
& q=\eta \\
& g=\frac{\eta}{A_{t}} \sqrt{\frac{\eta}{\frac{z_{3}}{\omega_{R}}-P_{e}}}
\end{aligned}
$$

One can already point out that if internal dynamic is not stable (or if q tightens towards infinity) then state variable $g$ will also strive towards infinity and vice versa. Even if $g$ converges, then $q$ also will converge. So, if it is unstable, the dynamic can be stabilized by a control which stabilizes state variable $\mathrm{g}$.

The derivate of internal variable allows us to acquire the equation of internal dynamics.

$\dot{\eta}=\dot{q}=\frac{1}{T_{W}}\left(1-\frac{q^{2}}{A_{t}^{2} g^{2}}\right)=\frac{1}{T_{W}}\left(1-\frac{\left(\frac{z_{3}}{\omega_{R}}-P_{e}\right)}{\eta}\right)$

With $\mathrm{P}_{\mathrm{e}}$ negative. 
The dynamic of the zero is determined by putting the external variables equal to zero. By considering these variables constants, it doesn't change final conclusion. Therefore, the dynamic of zero is:

$\dot{\eta}=f(\eta)=\frac{1}{T_{W}}\left(1+\frac{P_{e}}{\eta}\right)$

The point of equilibrium of this equation is $\eta_{0}=-P_{e}$

One can conclude on stability or instability of the dynamics of zero by examining the derivative of $\mathrm{f}(\eta)$ at the equilibrium point. If it is negative, the system is stable. If it is positive, the system is unstable.

$$
f^{\prime}\left(\eta_{0}\right)=-\frac{P_{e}}{T_{W} \eta_{0}^{2}}=\frac{1}{T_{W} \eta_{0}}>0
$$

The internal dynamic is therefore unstable. So, it can be concluded that the system is unstable in a closed loop. The next stage is to offer a control which stabilizes the system in a closed loop.

\subsection{In a Continuous Closed Loop Stability}

The main purpose of the closed loop study of the system is to determine the condition stability of the state variable $\mathrm{g}$. The stabilization of $\mathrm{g}$ will generate the stabilization of state variable $\mathrm{q}$ and consequently, the stabilization of internal dynamic. To this end, the expression of control is substitute in the dynamic equation of variable $\mathrm{g}$.

$$
\dot{g}=\frac{-1}{T_{g}} g+\frac{1}{T_{g}} u
$$

With:

$$
u=\frac{-A_{t}^{2} g^{3} T_{g}}{2 \omega_{R} q^{2}} v+\frac{3 T_{g}}{2 T_{w}}\left(1-\frac{q^{2}}{A_{t}^{2} g^{2}}\right) g+g \cdot q
$$

$v$ being a variant of the linear control equation (26) which will allow us to stabilize the system. After substitution of equation (41) in equation (40) and simplification, we obtain:

$$
\begin{aligned}
& \dot{g}=\left(-\frac{1}{T_{g}}+\frac{q}{T_{g}}+\frac{3}{2 T_{W}}\right) g-\frac{3 q^{2}}{2 T_{W} A_{t}^{2}} \cdot \frac{1}{g}-\frac{A_{t}^{2} g^{3}}{2 \omega_{R} q^{2}} v \\
& v=v_{1}-k_{4}\left(g-g_{r e f}\right)=-k_{1}\left(y-y_{r e f}\right)-k_{2}\left(\dot{y}-\dot{y}_{r e f}\right) \\
& -k_{3}\left(\ddot{y}-\ddot{y}_{r e f}\right)-k_{4}\left(g-g_{r e f}\right)
\end{aligned}
$$


The previous dynamic equation becomes:

$$
\begin{aligned}
& \dot{g}=m(g, q)= \\
& \left(-\frac{1}{T_{g}}+\frac{q}{T_{g}}+\frac{3}{2 T_{W}}\right) g- \\
& \frac{3 q^{2}}{2 T_{W} A_{t}^{2}} \cdot \frac{1}{g}-\frac{A_{t}^{2} g^{3}}{2 \omega_{R} q^{2}} v_{1}+\frac{A_{t}^{2} g^{3} k_{4}}{2 \omega_{R} q^{2}}\left(g-g_{r e f}\right)
\end{aligned}
$$

With $g_{\text {ref }}$ as constant.

It is then enough to choose $\mathrm{k}_{4}$ so that $\mathrm{g}$ is stable and converges towards $\mathrm{g}_{\mathrm{ref}}$. It is necessary to note that the choice of gains $\mathrm{k}_{1}, \mathrm{k}_{2}$ and $\mathrm{k}_{3}$ assures that $v_{1}$ will converge on zero.

To determine $\mathrm{k}_{4}$, equation (44) is linearized around a functioning point $\left(\mathrm{g}_{0}, \mathrm{q}_{0}\right)$ and the condition $\left.\frac{\partial m(g, q)}{\partial g}\right|_{(g 0, q 0)}<0$, is put.

$$
\begin{aligned}
& \left.\frac{\partial m(g, q)}{\partial g}\right|_{(g 0, q 0)}= \\
& \frac{-1}{T_{g}}+\frac{q_{0}}{T_{g}}+\frac{3}{2 T_{W}}+\frac{3 q_{0}^{2}}{2 T_{W} A_{t}^{2} g_{0}{ }^{2}} \\
& +\frac{A_{t}^{2}\left(4 g_{0}^{3}-3 g_{0}^{2} g_{r e f}\right) k_{4}}{2 \omega_{R} q_{0}{ }^{2}}
\end{aligned}
$$

To assure the stability of the system whichever the point of operation is, it is necessity to choose $\mathrm{k}_{4}$ according to the following relation:

$$
\begin{aligned}
& k_{4} \gg \\
& -\left(\frac{-1}{T_{g}}+\frac{q_{0}}{T_{g}}+\frac{3}{2 T_{W}}+\frac{3 q_{0}{ }^{2}}{2 T_{W} A_{t}^{2} g_{0}{ }^{2}}\right) \frac{2 \omega_{R} q_{0}{ }^{2}}{A_{t}^{2}\left(4 g_{0}{ }^{3}-3 g_{0}{ }^{2} g_{r e f}\right)}
\end{aligned}
$$

The very upper condition (>>) allows to make $\mathrm{k}_{4}$ independent of the point $\left(\mathrm{g}_{0}, \mathrm{q}_{0}\right)$

\subsection{Stabilizing Control}

The new linear control which stabilizes external and internal dynamics is therefore

$$
v=-k_{1}\left(y-y_{r f}\right)-k_{2}\left(\dot{y}-\dot{y}_{r f}\right)-k_{3}\left(\ddot{y}-\ddot{y}_{r e f}\right)-k_{4}\left(g-g_{r e f}\right)
$$

Gains $\mathrm{k}_{\mathrm{i}}(\mathrm{i}=0, \ldots, 4)$ are determined according to (31) and (46). 
In this work, we start with a first resolution in which a simplifying hypothesis was taken into consideration, this hypothesis is based on two models acquired after decoupling of the total system, and therefore the controlled system will be on the order of 7. The input of its control is the excitation voltage and its output is the terminal voltage. As shown, in sequence resolution, this system is linearized by the linearization method by return of state, and then stabilized by a linear order, and the effectiveness of such strategy will be tested by simulation.

\section{Simulation}

This test consists in forcing at zero the voltage across the generator or infinite bus for $100 \mathrm{~ms}$. The generator is initially charged to $60 \%$ at nominal power and not decoupled during the short-circuit from the network, which leads to important changes of the electric power. The role of power controller and rotor angle controller is to quickly maintain the terminal voltage and internal angle at their nominal values with minimum overshoot after the short-circuit.

Here, we test the performance of the proposed SISO controller, which was tested on the complete $7^{\text {th }}$ order model of the turbine-generator system in a single machine infinite bus. The parameters are chosen from [1]. The stability of the system which is validated by simulating a short-circuit at the secondary of the generator's transformer for a period of $100 \mathrm{~ms}$. We compare the performance of both the non-linear controller and the linear controller [5]. In Figures 2 to 5 we show the response of the terminal voltage $v_{t}$ after a short-circuit in which it is shown the stabilization of $v_{\mathrm{t}}$.

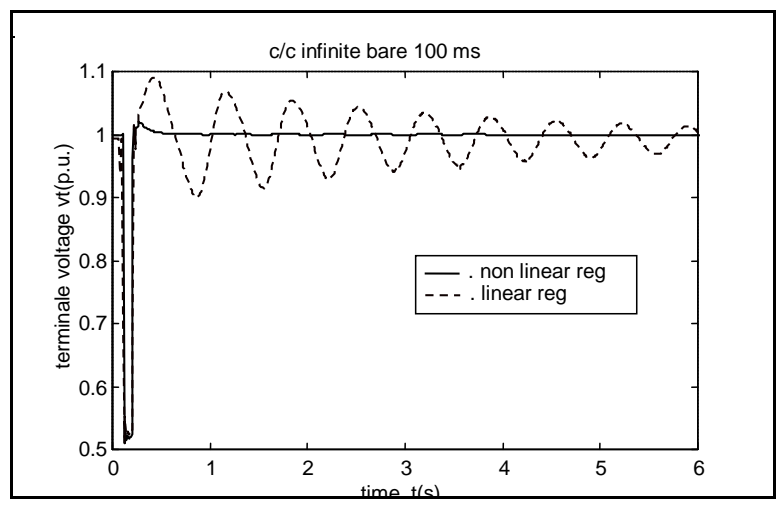

Figure 2

Terminal voltage $v_{\mathrm{t}}$ 


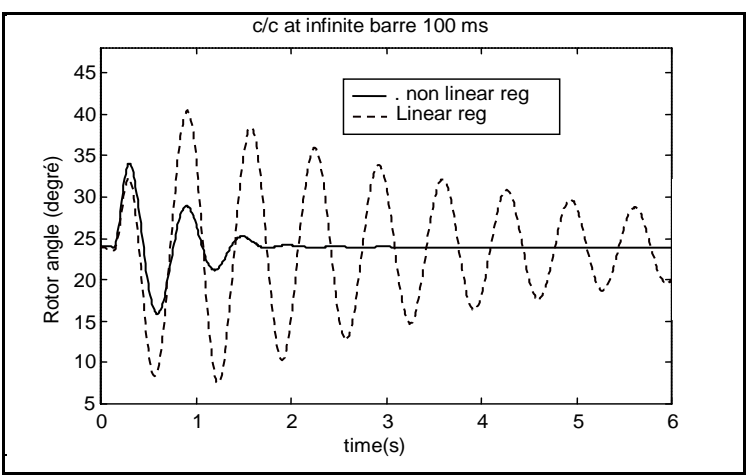

Figure 3

Rotor Angle $\delta$

After using the non-linear controller, it is observed that the dynamics of the rotor angle has a much shorter time for the oscillations. The same remarks are made for the Figures 4 and 5.

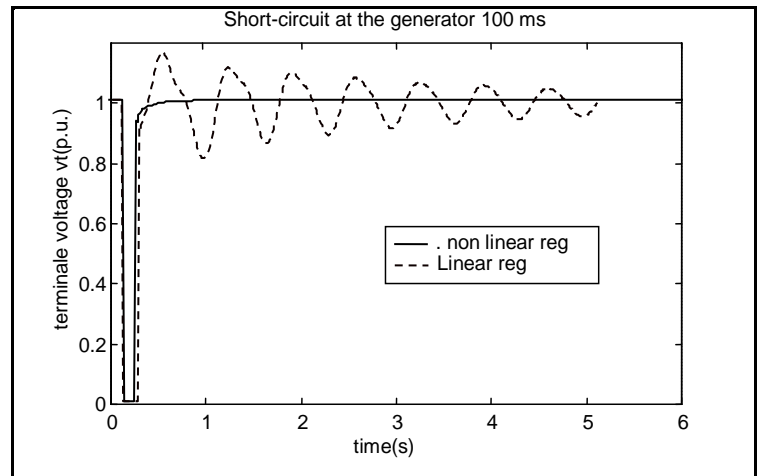

Figure 4

Terminal voltage $v_{\mathrm{t}}$

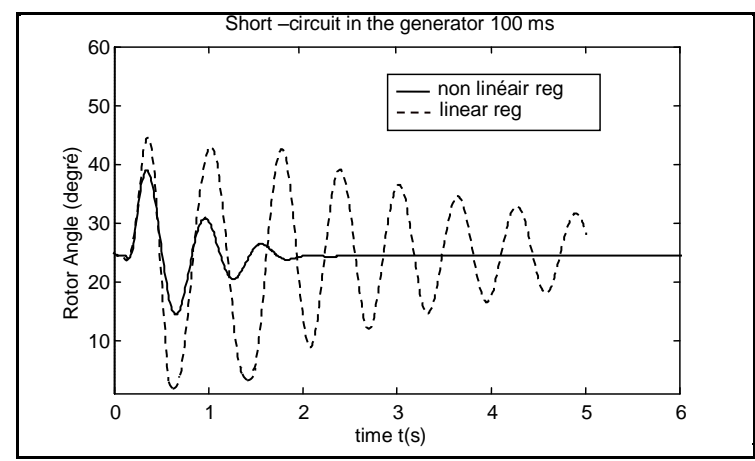

Figure 5

Rotor Angle $\delta$ 
After the short-circuit the bus frequency and the rotor angle of generator stabilized 2 to 4 times more rapidly and the terminal voltage came back at the first position twenty times more quickly than with the non-linear regulator. These performances assure for the network to resist at the important disturbance without losing it synchronism.

\section{Conclusion}

The considered machine in this work comprises synchronous generator and hydraulic turbine. We used for control the nonlinear regulator based on the feedback linearization. The controller is tested in simulation and compared with classical linear scheme. In conclusion, the controller is able to power system damping and the post fault regulation of the generator terminal voltage even after a large fault [6]. All these performance compensating nonlinear meet standard linear regulators are due to the fact that the linearization by feedback state ensures the validity of a priori linear control around the state space. The superiority of non-linear regulators is largely due to the inclusion of nonlinearities. However, the price is that with nonlinear control regulators efforts are bigger and the control laws are much more complex. Their implementation requires faster processors and are therefore more expensive [7], [8].

\section{References}

[1] Ouassima Akhrif, Francis-Aimé Okou, "Application of a Multivariable Feedback Linearization Scheme for Rotor Angle Stability and Voltage Regulation of Power Systems" IEEE Translations on Power Systems, Vol. 14, No. 2, May 1999

[2] Anderson, M and Fouad, A, "Power System Control and Stability", IEEE Press, 1993

[3] H. F. Wang, Y. S. Hao, "An Adaptative Power System Stabilizer Based on Turbine Governor Control" IEE $2^{\text {nd }}$ international conference on advences in power system control, operation and management, Dec. 1993, Hong Kong

[4] Do-Kwan Lee, Tae-Woong Yoon, “Adaptive Nonlinear Control of a Power System" Proceeding of the 1998 IEEE, International Conference on Control Applications, Triete, Italy, 1-4 Sept. 1998

[5] Okou, A. F. "Conception d'un regulateur non lineaire de tension et d'angle de charge pour un generateur synchrone", MS.Thesis, ETS, Montreal, 1996

[6] M. Labben ben Braick, F. Fnaiech, "Feedback Lenearizatin Control Technique Applied to a Three Phase Shnt Active Power Filter” IEEE, 2003

[7] Yang Han et al., "Control Strategies, Robustness Analysis, Digital Simulation and Practical Implementation for a Hybrid APF with a Resonant AC -link. ”Acta Polytechnica Hungarica, Vol. 7, N5, 2010

[8] Belabbes B, “Commande Non Linéare Robuste d'une Machine Synchrone a Aimants Permanents sans capteur Mécanique” Doctorat en ETT, Sidi Bel Abbes, Algérie 2007 